\title{
One Novel 2.43Kb Deletion and One Single Nucleotide Mutation of the INSR Gene in a Chinese Neonate with Rabson-Mendenhall Syndrome
}

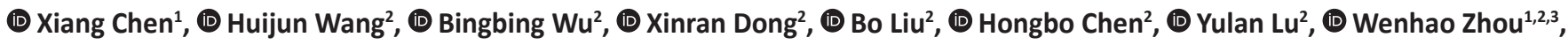 \\ (D) Lin Yang ${ }^{2,4}$ \\ ${ }^{1}$ Children's Hospital of Fudan University, Clinic of Neonatology, Shanghai, China \\ ${ }^{2}$ Children's Hospital of Fudan University, Key Laboratory of Birth Defects, Shanghai, China \\ ${ }^{3}$ Children's Hospital of Fudan University, Key Laboratory of Neonatal Diseases, Shanghai, China \\ ${ }^{4}$ Children's Hospital of Fudan University, Clinic of Endocrinology, Genetics and Metabolic Diseases, Shanghai, China
}

\begin{abstract}
What is already known on this topic?
Mutation of the insulin receptor gene is responsible for Rabson-Mendenhall syndrome (RMS) which is an autosomal recessive disorder. Typical symptoms of RMS include growth retardation, elfin face, gingival hyperplasia, acanthosis nigricans, hypertrichosis and insulin resistance.
\end{abstract}

\section{What this study adds?}

We report an atypical and mild RMS patient due to a compound heterozygosity consisting of a novel $2.43 \mathrm{~Kb}$ deletion and a known, pathogenic point mutation in the INSR gene.

\begin{abstract}
Mutations in the insulin receptor (INSR) gene are responsible for Donohue syndrome (DS) and Rabson-Mendenhall syndrome (RMS). Insulin resistance is a feature of both diseases.

Our patient was a Chinese neonate suffering from abnormal glucose homeostasis, hyperinsulinemia, dry skin, heavy hair, growth retardation and an elevated testosterone level. To search for candidate point mutations, small insertions or deletions and copy number variants, 2742 inherited disease-gene panel sequencing was performed. One pathogenic mutation (c.3355C > T, p.Arg1119Trp) and a novel 2.43 Kb deletion (chr19:7150507-7152938) in INSR were found. The patient was diagnosed as RMS. Sanger sequencing and real-time quantitative polymerase chain reaction (PCR) confirmed the missense variant and microdeletion, respectively. We therefore supposed that these variants were candidate mutations in this case. We report a novel $2.43 \mathrm{~Kb}$ deletion in INSR gene and provide further proof of the power of next generation sequencing in rare disease diagnosis.
\end{abstract}

Keywords: Insulin receptor gene, Rabson-Mendenhall syndrome, neonate, mutation, next generation sequencing

\section{Introduction}

Insulin receptor (INSR) is the gene responsible for a series of insulin resistance diseases, including hyperinsulinemic hypoglycemia, familial 5 [Online Mendelian Inheritance in Man (OMIM)\#609968], Donohue syndrome [(DS), also called leprechaunism; OMIM\#246200] and Rabson-Mendenhall syndrome [(RMS); OMIM\#262190]. The inheritance pattern of DS and RMS is autosomal recessive. Typical symptoms of DS include growth retardation, elfin face, gingival hyperplasia, acanthosis nigricans, hypertrichosis and insulin resistance (1). RMS and DS share similar symptoms. The symptoms of DS are more severe, have an infantile onset and may lead to early death. RMS is often encountered as 
being of childhood-onset and with survival up to adulthood with milder symptoms. The differential diagnosis is based on the onset age and severity of the disease (2).

In this study, we describe a Chinese, male, newborn with hyperinsulinemia and hyperglycemia. Next generation sequencing (NGS) detected a compound heterozygous mutations of INSR, including one known mutation and one novel $2.43 \mathrm{~Kb}$ deletion.

\section{Case Report}

The proband is a male infant and the first child of nonconsanguineous parents. During the fetal period, he was diagnosed with intrauterine growth retardation and oligohydramnios and was delivered by natural labor at
36 weeks of gestation with a low birth weight at $1.7 \mathrm{~kg}$. At age 13 days, he presented with dry skin and heavy hair over his whole body. The plantar grasp, Moro and sucking reflexes were weak. Facial malformation, abnormality of mouth size, acanthosis nigricans and abdominal distention were not observed. Clinical tests showed hyperglycemia (14.7 mmol/L), hyperinsulinemia (>300 IU/mL) and fasting hypoglycemia. C-peptide was $4.05 \mathrm{ng} / \mathrm{mL}(1.10-4.40 \mathrm{ng} /$ $\mathrm{mL}$ ). HbAlc (glycosylated hemoglobin) was normal (5.4\%). Other abnormal laboratory test results are shown in Table 1. Insulin auto-antibodies were negative. Routine blood tests, liver function tests and thyroid-stimulating hormone levels were normal. Echocardiography suggested a ventricular septal defect, an atrial septal defect and ultrasonography indicated swelling of both kidneys. Magnetic resonance imaging of the brain indicated an impaired myelination of

\begin{tabular}{|c|c|c|c|}
\hline & System or organ & Features & Proband \\
\hline \multirow{19}{*}{$\begin{array}{l}\text { Syndromic } \\
\text { features } \\
\text { of RMS } \\
\text { according to } \\
\text { OMIM }\end{array}$} & \multirow[t]{2}{*}{ Growth } & Intrauterine growth retardation & + \\
\hline & & Small for gestational age & $1.7 \mathrm{~kg}(\mathrm{GA}: 36 \mathrm{w})$ \\
\hline & Face & Coarse face, prognathism & - \\
\hline & Mouth & Large, fissured tongue; gingival hypoplasia; high-arched palate & - \\
\hline & Teeth & Dental dysplasia, premature eruption of teeth & - \\
\hline & Genitourinary & Large penis, clitoromegaly & - \\
\hline & \multirow[t]{2}{*}{ Skin } & Acanthosis nigricans, lichenified skin & - \\
\hline & & Dry skin & + \\
\hline & Nails & Onychauxis & - \\
\hline & Hair & Hypertrichosis c & + \\
\hline & \multirow{2}{*}{$\begin{array}{l}\text { Central nervous } \\
\text { system }\end{array}$} & Pineal hypertrophy & - \\
\hline & & Developmental delay & + \\
\hline & \multirow[t]{4}{*}{ Endocrine features } & Insulin resistant diabetes mellitus & - \\
\hline & & Diabetic ketoacidosis & - \\
\hline & & Altered melatonin secretion & 1 \\
\hline & & Precocious puberty & - \\
\hline & \multirow{3}{*}{$\begin{array}{l}\text { Laboratory } \\
\text { abnormalities }\end{array}$} & Postprandial hyperglycemia & + \\
\hline & & Fasting hypoglycemia & + \\
\hline & & Hyperinsulinemia & $>300 \mathrm{IU} / \mathrm{mL}$ \\
\hline \multirow{7}{*}{$\begin{array}{l}\text { Additional } \\
\text { positive test } \\
\text { results }\end{array}$} & \multirow{4}{*}{$\begin{array}{l}\text { Laboratory } \\
\text { abnormalities }\end{array}$} & Testosterone in plasma $(0.7-3.6 \mathrm{ng} / \mathrm{mL})$ & $18.2 \mathrm{ng} / \mathrm{mL}$ \\
\hline & & Urea in plasma $(2.5-6.5 \mathrm{mmol} / \mathrm{L})$ & $0.6 \mathrm{mmol} / \mathrm{L}$ \\
\hline & & Creatinine in plasma $(20-110 \mathrm{umol} / \mathrm{L})$ & $12 \mathrm{umol} / \mathrm{L}$ \\
\hline & & Hypokalemia (3.5-5.5 mmol/L) & $3.2 \mathrm{mmol} / \mathrm{L}$ \\
\hline & \multirow[t]{2}{*}{ Ultrasonography } & Ventricular septal defect, atrial septal defect & \\
\hline & & Swelling of both kidneys & \\
\hline & MRI & Impaired myelination of white matter & \\
\hline
\end{tabular}

+ : Patient owns this phenotype, -: This feature is absent in this patient, /: Whether this feature present in this patient is unknown,

GA: gestational age, OMIM: Online Mendelian Inheritance in Man, RMS: Rabson-Mendenhall syndrome, MRI: magnetic resonance imaging 
white matter. The mother had transient hypothyroidism during pregnancy. Otherwise the family history is negative. At the last outpatient follow-up at age four months, the patient's neurodevelopment was found to be delayed and that the high postprandial blod glucose $(>11 \mathrm{mmol} / \mathrm{L})$ and hyperinsulinemia (>300 IU/mL) persisted.

Pre-test counseling was performed by physicians and appropriate informed consent was signed by the patient's parents in the clinic. The criteria of genetic testing received approval from the ethics committees of the Children's Hospital, Fudan University (2016-235). Genomic DNA samples were extracted from whole blood using the

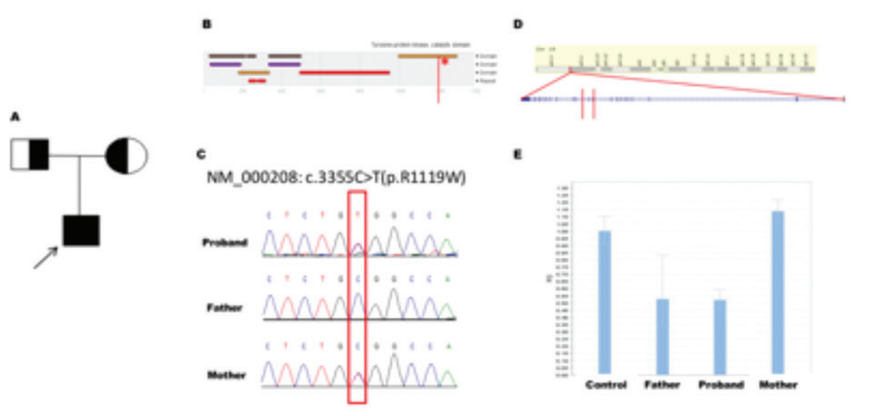

Figure 1. Insulin receptor gene compound heterozygous mutation: a known missense mutation and a novel microdeletion. A) Pedigree of the family. B) The SNV is located at the tyrosine-protein kinase catalytic domain and marked by red asterisk. C) Sanger sequencing shows the mutation is from mother. Insulin receptor gene locates at 19p13.319p13.2. D) The deletion fragment is marked within two red lines. This fragment contains Exon 11 and part of Exon 10. E) Real-time quantitative polymerase chain reaction shows that the deletion is from father

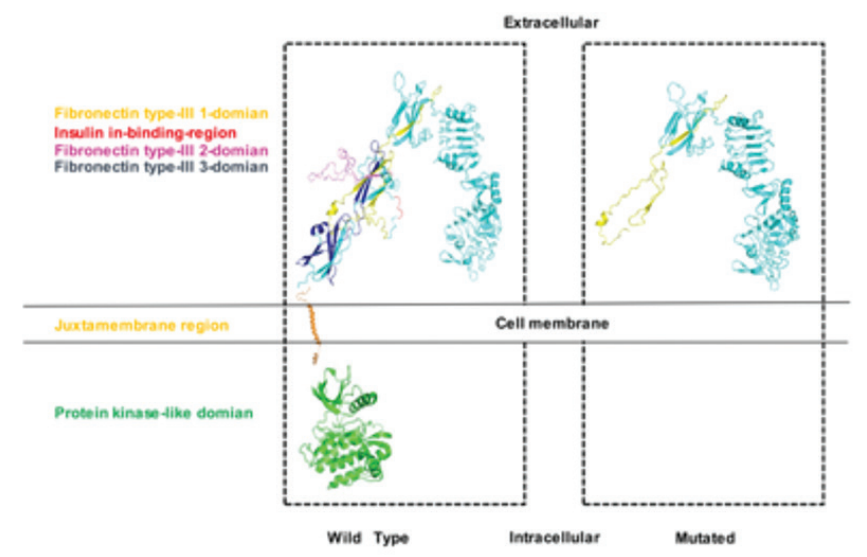

Figure 2. 3D structural modeling of insulin receptor protein. Comparing to the wild type, 3D structural modeling estimates a large portion of deficiency in the monomer form of the insulin receptor caused by the deletion. Different domains are marked by different colors with the color of the domain matched by the color of the domain name in the key
QIAamp DNA Blood Mini kit (QIAGEN, Germany) following the manufacturer's protocol. The quality and quantity of the DNA samples were measured using a NanoDrop 2000 spectrophotometer (Thermo Fisher Scientific, USA). Nucleic acid preparation and high-throughput sequencing were performed using standard protocols in a Clinical Laboratory Improvement Amendments (CLIA) compliant sequencing laboratory in Wuxi NEXTCODE (288 Fute Zhong Road, Waigaoqiao Free Trade Zone Shanghai 200131, China CLIA ID 99D2064856). Inherited-Disease panel sequencing was generated using the Agilent ClearSeq Inherited Disease kit, Illumina Cluster and SBS kit. The sequencing was performed using NGS on the Illumina Hiseq 2000/2500 platform. This covers a minimum of $98 \%$ of the genome with $20 \mathrm{X}$ coverage and was compared to a human reference sequence.

We identified a known-pathogenic mutation (c.3355C > T, p.Arg1119Trp) at exon 18 of the INSR gene (NM_000208). This mutation has been reported in a patient with DS (3). This mutation was recorded in HGMD (http://www.hgmd.cf.ac. uk/ac/index.php) as CM1119. The Exac database (http://exac. broadinstitute.org/) and the 1000 gene database (http://www. internationalgenome.org/) have no record of this variant. This mutation was also the only record in our internal database, which contains sequencing data of ClearSeq of 4071 patients. Paired primers were designed by using Primer3 website and primer-BLAST (5'-GGGAGGAGAACCCTGGTGAG-3' and '-ATCCGAGGAGGCCAGGAG-3'). Sanger sequencing indicated that this mutation was inherited from the mother (Figure $1 \mathrm{~A}, 1 \mathrm{~B}, 1 \mathrm{C})$.

We used CANOES (CNVs with an Arbitrary Number of Exome Samples) for basic detection of CNVs from NGS data at genelevel and region-level (4). Gene-level annotation was based on OMIM, Human Gene Mutation Database (HGMD), SwissProt and RefSeq. Region-level information was annotated by Database of Genomic Variants (DGV), and Database of Genomic Variation and Phenotype in Humans using Ensembl Resources (DECIPHER). We detected a novel deletion of approximately $2.43 \mathrm{~Kb}$ (chr 19:7150507-7152938) within the INSR gene (Figure 1D). This deletion is not found in HGMD, DECIPHER or DGV. Additionally, it is absent from our internal database. The detected mutation was confirmed using realtime quantitative PCR. PCR-amplified DNA products were subjected to direct automated sequencing (ABI step one plus v.2.0). Both strands of each amplicon were sequenced using the primers 5'-CCTGACCTGGGGACGAAAA-3' and 5'-GTCTCCACCATTCGAGTCTGA-3'. Real-time quantitative PCR indicated the deletion was from the father (Figure 1E). This region covers part of exon 10 and all of exon 11 . The deletion is estimated to cause a truncated protein. We performed a three-dimensional (3D) structural modeling of 
the monomer form of the INSR and mapped the deletion on to it. The PBD number of the INSR extracellular region is $4 Z X B$.E, that of the juxtamembrane region is 2MFR.A and that of the tyrosine kinase domain is 3BU3.A (5). The 3D structural modeling shows the monomer form of the INSR. The Fibronectin type-III 2-domain, Fibronectin typeIII 3-domain, Insulin in-binding-region, protein kinase-like domain, juxtamembrane region and partial Fibronectin type-III 1-domain are absent (Figure 2) (5).

\section{Discussion}

In this Chinese newborn baby, we identified a novel microdeletion and a known missense mutation within the INSR gene, which caused a compound heterozygous mutation. The INSR gene is located at chromosome 19 and encodes the INSR. HGMD contains 178 mutations of INSR. For RMS, 26 mutations of INSR are reported. Of these, two compound heterozygous mutations, each containing one deletion and one single nucleotide mutation, have been previously reported. A gross deletion containing exons 9 and 10 was reported in a 15-year-old RMS patient (6). This patient carried a mutation (p.Ser635Leu) in INSR with a compound heterozygous genotype. The main phenotypes are hyperglycemia and hyperinsulinemia. Another gross deletion contains exon 18. This RMS patient, as a compound heterozygote, also carried a mutation (p.Val66Ala). Nephrocalcinosis was found to be one of the patient's dominant features (7). For the patient we report, the missense mutation (c.3355C > T, p.R1119W) has been reported from a DS patient who had symptoms at birth and died at three months of age (3). Our patient had symptoms 13 days after birth. Some typical RMS features of this patient include abnormal glucose homeostasis, hyperinsulinemia, dry skin, thick hair, elevated testosterone and growth retardation. We diagnosed this patient as RMS. With a microdeletion, our patient presented a mild and atypical phenotype, which may be explained by the unclear genotype-phenotype correlation of mutations in INSR. Different missense mutations in the same codon relate to different phenotypes (8). One DS patient, bearing a homozygous deletion in INSR resulting in inactivation of the INSR lived for 3.5 years (9). The coexistence of modifier genes and compensatory pathways may explain the phenotypic variability (10).

The insulin receptor is a tetramer of two $\alpha$ monomers and two $\beta$ monomers. It is widely expressed and plays a vital role as a mediator between the extracellular and intracellular insulin signaling pathway. The whole region of the $\alpha$-subunit is extracellular. The $\alpha$-subunit contains a Leu- rich-compositionally biased region, Cys-rich-compositionally biased region, a Fibronectin type-III 1-domain and an insulin in-binding-region. The $\beta$-subunit extends through the cell membrane into the cytoplasm. The extracellular region of the $\beta$-subunit contains a Fibronectin type-III 2 -domain and a Fibronectin type-III 3-domain. The cytoplasmic region of the $\beta$-subunit consists of several functional domains including a juxtamembrane region, a tyrosine kinase domain and the carboxy-terminal-region $(5,11)$. The $\alpha$-subunit is responsible for binding affinity to insulin. The Cys-rich-compositionally biased region is the main binding site of insulin. Fibronectin type-III domains 1 and 2 form the secondary insulin-binding site (5). Deficiency of the juxtamembrane region makes the folding of IR unstable and affects its downstream processing (12). The function of the Fibronectin type-III 3-domain remains unknown. A helical transmembrane region follows the Fibronectin type-III 3-domain. Through binding with insulin, IR initiates the phosphorylation of different phosphotyrosine residues in the tyrosine kinase domain (13). Downstream IR substrates bind to phosphotyrosine residues of IR and regulate two main signaling pathways: the phosphatidylinositol 3-kinase-AKT/protein kinase B (PI3K-AKT/PKB) pathway and the Ras-MAPK pathway. The PI3K-AKT/PKB pathway is responsible for controlling cell growth and differentiation. The metabolic action of insulin is mainly regulated by the RasMAPK pathway (14). 3D structural modeling indicates that with deficiencies in both $\alpha$-subunit and $\beta$-subunit, IR may be unable to combine with insulin receptor substrates and recruit the downstream signaling molecules (15).

Our patient did not show some of the typical features of RMS including coarse face, gingival hyperplasia and acanthosis nigricans. These three symptoms can be absent in neonates and may develop in adolescence (16). The insulin receptor is expressed in the heart and regulates cardiac cell activity though the PI3K-AKT pathway. Insulin receptor deficiency possibly leads to cardiac dysfunction, as observed in some patients $(10,17)$ and proven using animal models (18). There is no evidence indicating any relationship between heart structural malformation and insulin receptors, so we consider that the ventricle septal defect is not a consequence of insulin receptor deficiency in this case. The long-term prognosis of RMS patients is poor (19). Recombinant human insulin-like growth factor 1 and recombinant leptin are recommended for treatment of severe insulin resistance syndrome $(19,20)$. However, the complications and safety of these drugs remain unknown (21)

In summary, we show an RMS patient carrying one known pathogenic mutation and one novel deletion in INSR. Since the presenting clinical features of patients with insulin resistance syndrome can be atypical, when the diagnosis 
is in doubt genetic testing may help to identify the final diagnosis.

\section{Acknowledgments}

The authors would like to express their gratitude to the participating family. This work was supported by grants from Leading Talents ground of Shanghai Municipal Human Resources and Social security bureau (2014-738), Science and Technology Commission of Shanghai Municipal (14411950402) and National Natural Science Foundation of China (81401010).

\section{Ethics}

Informed Consent: The samples used in this study were collected with the appropriate informed consent and approval of the ethics committee of Children's Hospital, Fudan University. The methods used in this study were performed in accordance with the approved guidelines.

Peer-review: External and internal peer-reviewed.

\section{Authorship Contributions}

Surgical and Medical Practices: Lin Yang, Concept: Lin Yang, Wenhao Zhou, Design: Lin Yang, Wenhao Zhou, Xiang Chen, Data Collection or Processing: Bo Liu, Yulan Lu, Hongbo Chen, Analysis or Interpretation: Xinran Dong, Huijun Wang, Bingbing Wu, Literature Search: Xiang Chen, Writing: Xiang Chen.

Financial Disclosure: The authors have indicated that they have no financial relationships relevant to this article to disclose.

\section{References}

1. Nijim Y, Awni Y, Adawi A, Bowirrat A. Classic Case Report of Donohue Syndrome (Leprechaunism; OMIM *246200): The Impact of Consanguineous Mating. Medicine (Baltimore) 2016;95:e2710.

2. Grasso V, Colombo C, Favalli V, Galderisi A, Rabbone I, Gombos S, Bonora E, Massa O, Meschi F, Cerutti F, Iafusco D, Bonfanti R, Monciotti C, Barbetti F. Six cases with severe insulin resistance (SIR) associated with mutations of insulin receptor: Is a Bartter-like syndrome a feature of congenital SIR? Acta Diabetol 2013;50:951-957. Epub 2013 Jul 4

3. Desbois-Mouthon C, Girodon E, Ghanem N, Caron M, Pennerath A, Conteville P, Magre J, Besmond C, Goossens M, Capeau J, Amselem S. Molecular analysis of the insulin receptor gene for prenatal diagnosis of leprechaunism in two families. Prenat Diagn 1997;17:657-663.

4. Backenroth D, Homsy J, Murillo LR, Glessner J, Lin E, Brueckner M, Lifton R, Goldmuntz E, Chung WK, Shen Y. CANOES: detecting rare copy number variants from whole exome sequencing data. Nucleic Acids Res 2014;42:e97. Epub 2014 Apr 25

5. Berman HM, Westbrook J, Feng Z, Gilliland G, Bhat TN, Weissig H, Shindyalov IN, Bourne PE. The Protein Data Bank. Nucleic Acids Res 2000;28:235-242.

6. Brown RJ, Cochran E, Gorden P. Metreleptin improves blood glucose in patients with insulin receptor mutations. J Clin Endocrinol Metab 2013;98:1749-1756. Epub 2013 Aug 22
7. Simpkin A, Cochran E, Cameron F, Dattani M, de Bock M, Dunger DB, Forsander G, Guran T, Harris J, Isaac I, Hussain K, Kleta R, Peters C, Tasic V, Williams R, Yap Kok Peng F, O'Rahilly S, Gorden P, Semple RK, Bockenhauer D. Insulin Receptor and the Kidney: Nephrocalcinosis in Patients with Recessive INSR Mutations. Nephron Physiol 2014;128:5556. Epub 2014 Oct 24

8. Ardon O, Procter M, Tvrdik T, Longo N, Mao R. Sequencing analysis of insulin receptor defects and detection of two novel mutations in INSR gene. Mol Genet Metab Rep 2014;1:71-84.

9. Wertheimer E, Lu SP, Backeljauw PF, Davenport ML, Taylor SI. Homozygous deletion of the human insulin receptor gene results in leprechaunism. Nat Genet 1993;5:71-73.

10. Falik Zaccai TC, Kalfon L, Klar A, Elisha MB, Hurvitz H, Weingarten G, Chechik E, Fleisher Sheffer V, Haj Yahya R, Meidan G, Gross-Kieselstein E, Bauman D, Hershkovitz S, Yaron Y, Orr-Urtreger A, Wertheimer E. Two novel mutations identified in familial cases with Donohue syndrome. Mol Genet Genomic Med 2014;2:64-72. Epub 2013 Nov 14

11. Hubbard SR. The insulin receptor: both a prototypical and atypical receptor tyrosine kinase. Cold Spring Harb Perspect Biol 2013;5:a008946.

12. Siala-Sahnoun O, Dhieb D, Ben Thabet A, Hmida N, Belguith N, Fakhfakh F. First molecular diagnosis of Donohue syndrome in Africa: novel unusual insertion/deletion mutation in the INSR gene. Mol Biol Rep 2016;43:165-173. Epub 2016 Feb 13

13. Hubbard SR. Crystal structure of the activated insulin receptor tyrosine kinase in complex with peptide substrate and ATP analog. EMBO J 1997;16:5572-5581.

14. Leavens KF, Birnbaum MJ. Insulin signaling to hepatic lipid metabolism in health and disease. Crit Rev Biochem Mol Biol 2011;46:200-215. Epub 2011 Apr 5

15. Longo N, Singh R, Griffin LD, Langley SD, Parks JS, Elsas LJ. Impaired growth in Rabson-Mendenhall syndrome: lack of effect of growth hormone and insulin-like growth factor-I. J Clin Endocrinol Metab 1994;79:799-805

16. Wei C, Burren CP. Diagnostic and management challenges from childhood, puberty through to transition in severe insulin resistance due to insulin receptor mutations. Pediatr Diabetes 2017;18:835-838. Epub 2017 Jan 17

17. Nobile S, Semple RK, Carnielli VP. A novel mutation of the insulin receptor gene in a preterm infant with Donohue syndrome and heart failure. J Pediatr Endocrinol Metab 2012;25:363-366.

18. Marchand A, Atassi F, Mougenot N, Clergue M, Codoni V, Berthuin J, Proust C, Trégouët DA, Hulot JS, Lompré AM. miR-322 regulates insulin signaling pathway and protects against metabolic syndrome-induced cardiac dysfunction in mice. Biochim Biophys Acta 2016;1862:611-621. Epub 2016 Jan 13

19. Musso C, Cochran E, Moran SA, Skarulis MC, Oral EA, Taylor S, Gorden P. Clinical course of genetic diseases of the insulin receptor (type A and Rabson-Mendenhall syndromes): a 30-year prospective. Medicine (Baltimore) 2004;83:209-222.

20. de Kerdanet M, Caron-Debarle M, Nivot S, Gaillot T, Lascols O, Fremont B, Bonnaure-Mallet M, Gie S, Massart C, Capeau J. Ten-year improvement of insulin resistance and growth with recombinant human insulin-like growth factor 1 in a patient with insulin receptor mutations resulting in leprechaunism. Diabetes Metab 2015;41:331337. Epub 2014 Nov 25

21. Weber DR, Stanescu DE, Semple R, Holland C, Magge SN. Continuous subcutaneous IGF-1 therapy via insulin pump in a patient with Donohue syndrome. J Pediatr Endocrinol Metab 2014;27:1237-1241. 